\title{
OPEN Perinatal depression and anxiety of primipara is higher than that of multipara in Japanese women
}

\author{
Yukako Nakamura ${ }^{1}$, Takashi Okada ${ }^{2}$, Mako Morikawa ${ }^{2}$ Aya Yamauchi $^{3}$, Maya Sato ${ }^{1}$, \\ Masahiko Ando ${ }^{4}$ \& Norio Ozaki ${ }^{1,2}$
}

The proportion of women who experience a depressive state after delivery differs between primiparas and multiparas, so it is important to clarify the different factors related to depression between the two groups. In this study, we confirmed the differences in depressive states, the perinatal period, and social support between primiparas and multiparas, and clarified their characteristics. Data were extracted from a prospective cohort questionnaire survey conducted on pregnant women in Japan that included sociodemographic questions, the Edinburgh Postnatal Depression Scale, and the Japanese version of the Social Support Questionnaire. We carried out the chi-square test, Student's $t$-test, and analysis of covariance to compare responses between primiparas and multiparas. A total of 1138 primiparas and 380 multiparas provided valid responses. We found that primiparas had higher rates of experiencing maternity blues and postpartum depression than multiparas. We also found that primiparas had higher anxiety scores than multiparas. Primiparas with postpartum depression perceived a lower number of persons available to provide social support than primiparas without postpartum depression. These findings suggest that it is important to provide pregnant women, especially for primiparas, with information that allows them to increase the number of people who can provide them with support.

Many mothers experience a large physical and psychosocial burden in the perinatal period, which can lead to a high risk of developing depression ${ }^{1}$. It is estimated that approximately $10-15 \%$ of mothers experience perinatal depression ${ }^{2-5}$, which has similar symptoms to major depressive disorder (MDD). In our previous study, we reported that $6.6 \%$ of pregnant women showed persistent depression during pregnancy to the postpartum period, and $10.4 \%$ showed depression for the first time after childbirth ${ }^{6}$.

The proportion of those who experience a depressed state after delivery differs between primiparas and multiparas $^{7-9}$. In Japan, several studies have reported that the rate of women with depression after childbirth is higher for primiparas than multiparas ${ }^{10-12}$ and being a primipara has been proposed to be a risk factor for perinatal depression. In studies of non-Japanese subjects, it has been reported that being a multipara is a risk factor for postpartum depression (PPD $)^{13-15}$. On a global scale, the prevalence of PPD differs by country and is greater than previously thought ${ }^{16}$. Therefore, it is important to clarify PPD characteristics and risk factors for each nation.

Mothers with maternity blues $(\mathrm{MB})$ experience transient mood swings that usually subside within days after childbirth $^{17-19}$. The prevalence rates of MB reported in the literature vary from 40 to $80 \%^{20}$. Based on a British study that used Stein's Maternity Blues Scale (Stein's Scale), $67 \%$ of postpartum women are diagnosed with $\mathrm{MB}^{21}$. However, studies in Japan have reported a prevalence of $15-35 \%{ }^{22-25}$. Although MB is transitional, it is a highly prevalent mental health problem ${ }^{26}$. We previously reported that $\mathrm{MB}$ could be a risk factor for $\mathrm{PPD}^{6}$.

We also reported that social support has a protective function against a postpartum depressed state ${ }^{27}$. On the other hand, although support for mothers at high risk of PPD is useful, it has also been reported that support programs do not change mental health outcomes in mothers at low risk of PPD $^{28}$. Social support is a limited

\footnotetext{
${ }^{1}$ Department of Psychiatry, Nagoya University Graduate School of Medicine, 65 Tsurumai-cho, Showa-ku, Nagoya, Aichi 466-8550, Japan. ${ }^{2}$ Department of Child and Adolescent Psychiatry, Nagoya University Graduate School of Medicine, 65 Tsurumai-cho, Showa-ku, Nagoya, Aichi 466-8550, Japan. ${ }^{3}$ Psychiatry/Child and Adolescent Psychiatry, Nagoya University Hospital, 65 Tsurumai-cho, Showa-ku, Nagoya, Aichi 466-8550, Japan. ${ }^{4}$ Department of Advanced Medicine, Data Coordinating Center, Nagoya University Hospital, 65 Tsurumai-cho, Showa-ku, Nagoya, Aichi 466-8550, Japan. ${ }^{\square}$ email: okada@med.nagoya-u.ac.jp
} 


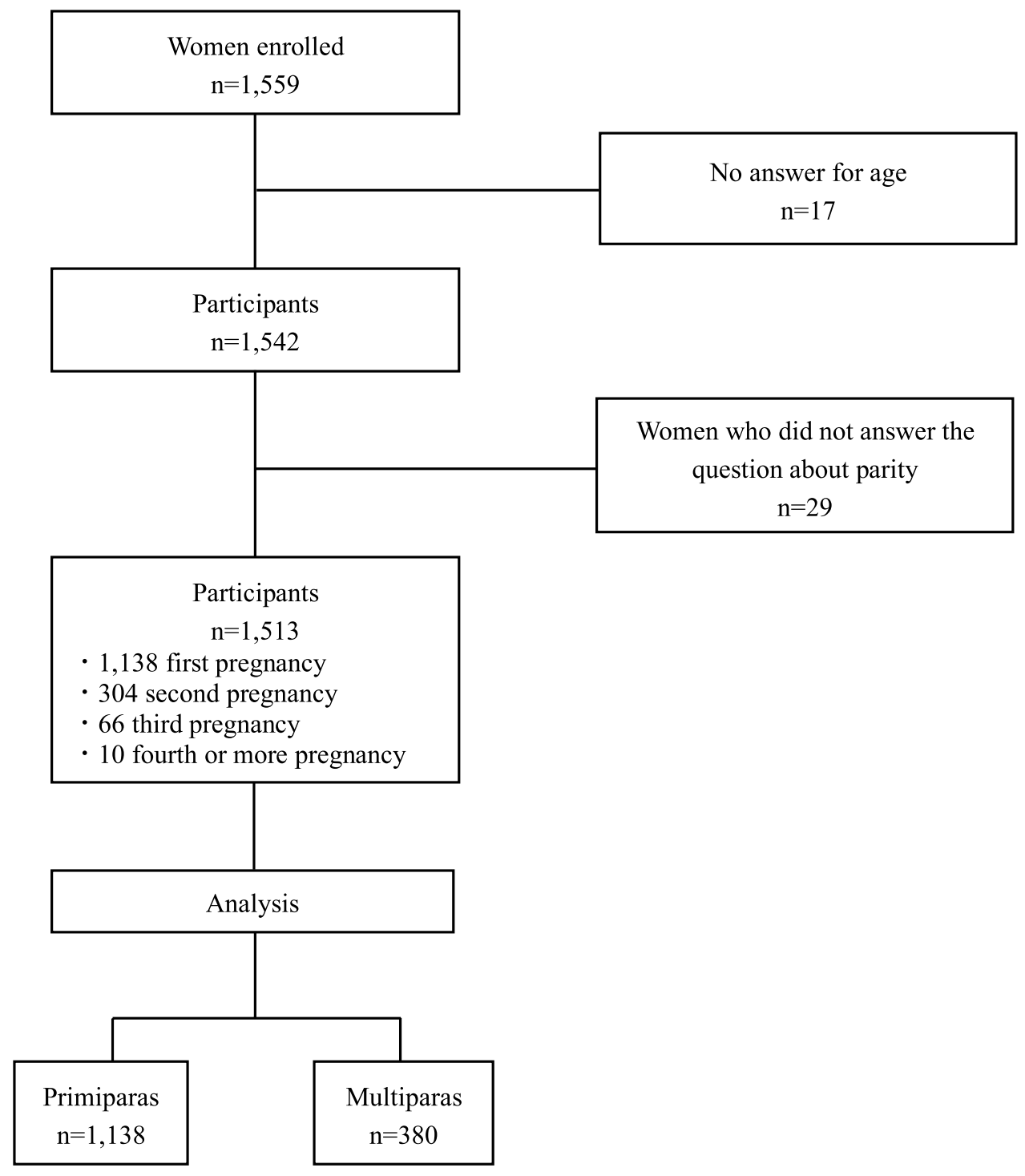

Figure 1. Flowchart of the recruitment process.

resource, and it is important to identify groups that need more social support and groups that could experience a positive change with social support in order to make effective use of limited resources.

The differences in MB and social support by parity and their impact on PPD are unclear. In order to provide effective support for depressive maternal women, it is important to confirm the differences in social support and depressive states in the perinatal period between primiparas and multiparas and to clarify their characteristics. In this study, we focused our attention on parity and compared the proportion of primiparas and multiparas presenting with depressive state and $\mathrm{MB}$ in the perinatal period. In addition, we clarified the characteristics of Japanese primiparas considered at high risk of PPD.

\section{Results}

In total, 1559 women submitted valid questionnaire responses at around the 25 th week of pregnancy. The average age of the participants was $32.4 \pm 4.6$ years $(n=1542)$. A total of 1138 women were recruited for this study during their first pregnancy, 304 women during their second pregnancy, 66 women during their third pregnancy, and 10 women during their fourth or more pregnancy. Forty-six women did not answer the question about age or parity. Therefore, this study included 1138 primiparas and 380 multiparas. A flowchart of the recruitment process is shown in Fig. 1. A comparison of the characteristics of primiparas and multiparas is shown in Table 1.

Descriptive statistics were used to calculate means and percentages for participant characteristics and chisquare tests and Student's $t$-tests were used to compare sociodemographic information based on parity (Table 1). Multiparas were significantly older than primiparas $\left(p=3.0 \times 10^{-8}\right.$, Student's t-test). Using a previously described method $^{29}$, the history of MDD was calculated based on the Inventory to Diagnose Depression, Lifetime version (IDDL). There was no significant difference in the rate of prior history of MDD or presence of mental disease between primiparas and multiparas ( $p=0.450$ and 0.595 , chi-square test). There was also no significant difference 


\begin{tabular}{|c|c|c|c|c|c|}
\hline & \multicolumn{2}{|c|}{ Primiparas $(\mathrm{N}=1138)$} & \multicolumn{2}{|c|}{ Multiparas $(\mathrm{N}=\mathbf{3 8 0})$} & \multirow{2}{*}{\begin{tabular}{|l|} 
Chi-square test \\
$p$-value \\
\end{tabular}} \\
\hline & $\mathbf{n}$ & $\%$ & $\mathbf{n}$ & $\%$ & \\
\hline \multicolumn{6}{|l|}{ IDDL } \\
\hline Negative & 492 & 72.0 & 121 & 69.1 & \\
\hline Positive & 191 & 28.0 & 54 & 30.9 & 0.450 \\
\hline \multicolumn{6}{|c|}{ Presence of mental disease } \\
\hline Negative & 493 & 83.7 & 135 & 85.4 & \\
\hline Positive & 96 & 16.3 & 23 & 14.6 & 0.595 \\
\hline \multicolumn{6}{|l|}{ Baby's sex } \\
\hline Boy & 318 & 50.8 & 85 & 52.1 & \\
\hline \multirow[t]{3}{*}{ Girl } & 308 & 49.2 & 78 & 47.9 & 0.759 \\
\hline & \multicolumn{2}{|c|}{ Primiparas $(\mathrm{N}=1138)$} & \multicolumn{2}{|c|}{ Multiparas $(\mathrm{N}=\mathbf{3 8 0})$} & Student's $t$-test \\
\hline & $\mathbf{n}$ & Mean \pm SD & $\mathbf{n}$ & Mean \pm SD & $p$-value ${ }^{\mathrm{b}}$ \\
\hline Age (years) & 1133 & $32.0 \pm 4.7$ & 380 & $33.5 \pm 4.1$ & $3.0 \times 10^{-8}$ \\
\hline Partner's age(years) & 597 & $35.1 \pm 6.0$ & 161 & $36.0 \pm 5.4$ & 0.058 \\
\hline Education years & 600 & $15.2 \pm 2.0$ & 161 & $14.9 \pm 2.1$ & 0.143 \\
\hline Baby's weight & 623 & $2995.1 \pm 410.2$ & 160 & $3001.7 \pm 356.5$ & 0.851 \\
\hline
\end{tabular}

Table 1. Comparison of characteristics of primiparas and multiparas. IDDL, Inventory to Diagnose

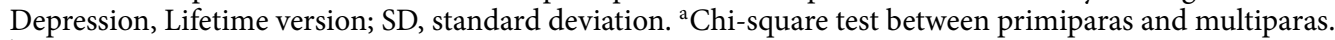
bstudent's $t$-test between primiparas and multiparas.

in years of education, partner's age, baby's weight ( $p=0.143,0.058$ and 0.851 , Student's $t$-test), or baby's sex $(p=0.759$, chi-square test $)$ among primiparas and multiparas. The main reason for missing values was differences in the time of information collection. The IDDL started to be conducted from April 2011, and data on years of education, presence or absence of mental disease, partner's age, and baby's sex and weight started to be collected from June 2012.

The prevalence rates of PPD based on the Edinburgh Postnatal Depression Scale (EPDS) score at four time points (T1-T4) and MB based on Stein's Scale at five time points (1, 2, 3, 4, and 5 days after delivery) for primiparas and multiparas are shown in Table 2. Means and standard deviations regarding responses to the EPDS were $24.3 \pm 6.8$ gestational weeks for $\mathrm{T} 1$, and $36.0 \pm 0.9$ gestational weeks for $\mathrm{T} 2,5.0 \pm 0.6$ days after delivery for $\mathrm{T} 3$, and $32.1 \pm 5.2$ days after delivery for T4. For the EPDS scores, the Bonferroni correction was performed by multiplying the number of test repetitions (four time points) by the $p$-value of the chi-square test, and for Stein's Scale scores, the Bonferroni correction was performed by multiplying the number of test repetitions (five time points) by the $p$-value of the chi-square test (Table 2). We confirmed the difference between the non-depressive group and the PPD group using the chi-square test.

In primiparas, the ratio of PPD was high. Next, we confirmed the difference in MB between the negative group and the positive group using the chi-square test. In primiparas, the ratio of MB was high (Table 2).

We show the results regarding the association of PPD and MB with age in Table 3 . The mean age did not significantly differ between the non-depressive and PPD groups or between the negative and positive of MB groups ( $p=1.000$ and 0.172 , Student's $t$-test). We confirmed that age had no influence on depression during pregnancy or the postpartum period, or on MB in this analysis. However, primiparas and multiparas showed a significant difference in age (Table 1); therefore, in our subsequent analyses (Tables 4, 5,6) we chose a method that strictly excluded the influence of age. First, we performed the parallel test and confirmed that there was no interaction between the factor and the covariate. Next, we performed an analysis of covariance (ANCOVA) with age as a covariate.

Table 4 shows the EPDS scores from pregnancy to the postpartum period. Primiparas had a significantly higher Anxiety score than did multiparas at T1, T3, and T4 ( $p=0.011,5.0 \times 10^{-5}$ and $9.1 \times 10^{-9}$, ANCOVA).

A comparison of score on the Japanese version of the Social Support Questionnaire (J-SSQ) between primiparas and multiparas is shown in Table 5. Primiparas had higher "Number of Persons" (NP) scores than did multiparas at T1 and T2, and higher "Satisfaction Rating" (SR) scores at T1. The main reason for missing values was differences in the time of information collection, as we started the evaluation of J-SSQ scores during early pregnancy in August 2004, and that of 1 month after birth in April 2006.

Table 6 shows the results of J-SSQ scores in primiparas in the PPD group and non-depressive group. The PPD group had significantly lower postpartum NP scores than the non-depressive group $(p=0.011$, ANCOVA).

\section{Discussion}

We found that primiparas had a higher rate of experiencing MB and higher rates of PPD compared with multiparas. The rate of Japanese women who experience MB is reported to be $15-35 \%{ }^{22-25}$. According to the results of the present study, the percentage of $\mathrm{MB}$ in primiparas (43.0\%) is significantly higher than that in multiparas (25.9\%). In addition, the rate of primiparas (15.2\%) who experience PPD is higher than that of multiparas (6.7\%). These results are consistent with previous reports for Japanese women, indicating that being primipara is a risk 


\begin{tabular}{|c|c|c|c|c|c|c|c|}
\hline & \multicolumn{2}{|c|}{ Primiparas } & \multicolumn{2}{|c|}{ Multiparas } & \multirow{2}{*}{\multicolumn{3}{|c|}{\begin{tabular}{|l|} 
Chi-square test \\
$p$-value \\
\end{tabular}}} \\
\hline & n & $\%$ & $\mathbf{n}$ & $\%$ & & & \\
\hline \multicolumn{8}{|l|}{ EPDS $^{\mathrm{a}}$} \\
\hline T1 score $\leq 8$ & 915 & 81.0 & 306 & 81.8 & & & \\
\hline T1 score $\geq 9$ & 214 & 19.0 & 68 & 18.2 & \multicolumn{3}{|l|}{1.000} \\
\hline T2 score $\leq 8$ & 867 & 85.2 & 293 & 86.4 & & & \\
\hline T2 score $\geq 9$ & 151 & 14.8 & 46 & 13.6 & \multicolumn{3}{|l|}{1.000} \\
\hline T4 score $\leq 8$ & 790 & 77.5 & 291 & 83.9 & & & \\
\hline T4 score $\geq 9$ & 229 & 22.5 & 56 & 16.1 & \multicolumn{3}{|l|}{0.048} \\
\hline T5 score $\leq 8$ & 794 & 76.9 & 300 & 86.2 & & & \\
\hline T5 score $\geq 9$ & 238 & 23.1 & 48 & 13.8 & \multicolumn{3}{|l|}{$9.0 \times 10^{-4}$} \\
\hline \multicolumn{8}{|l|}{ Stein's scale $^{\mathrm{b}}$} \\
\hline Dayl score $\leq 7$ & 823 & 82.4 & 304 & 89.1 & & & \\
\hline Dayl score $\geq 8$ & 178 & 17.6 & 37 & 10.9 & \multicolumn{3}{|l|}{0.016} \\
\hline Day2 score $\leq 7$ & 828 & 81.8 & 302 & 88.0 & & & \\
\hline Day2 score $\geq 8$ & 184 & 18.2 & 41 & 12.0 & \multicolumn{3}{|l|}{0.037} \\
\hline Day3 score $\leq 7$ & 823 & 81.3 & 311 & 90.4 & & & \\
\hline Day3 score $\geq 8$ & 189 & 18.7 & 33 & 9.6 & \multicolumn{3}{|l|}{$4.2 \times 10^{-4}$} \\
\hline Day4 score $\leq 7$ & 809 & 80.2 & 310 & 89.9 & & & \\
\hline Day4 score $\geq 8$ & 200 & 19.8 & 35 & 10.1 & \multicolumn{3}{|l|}{$2.1 \times 10^{-4}$} \\
\hline Day5 score $\leq 7$ & 831 & 82.1 & 308 & 88.8 & & & \\
\hline \multirow[t]{3}{*}{ Day5 score $\geq 8$} & 181 & 17.9 & 39 & 11.2 & \multicolumn{3}{|l|}{0.019} \\
\hline & \multicolumn{2}{|c|}{ Primiparas } & \multicolumn{2}{|c|}{ Multiparas } & Chi-square test & \multirow[b]{2}{*}{ OR } & \multirow[b]{2}{*}{ CI $(95 \%)$} \\
\hline & $\mathbf{n}$ & $\%$ & $\mathbf{n}$ & $\%$ & $p$-value ${ }^{\mathrm{d}}$ & & \\
\hline \multicolumn{8}{|l|}{ Depressive group } \\
\hline Non-depressive group & 646 & 84.8 & 236 & 93.3 & & & \\
\hline Postpartum depressive group & 116 & 15.2 & 17 & 6.7 & 0.002 & 2.09 & $1.33-3.31$ \\
\hline \multicolumn{8}{|l|}{ Maternity blues } \\
\hline Negative group & 564 & 57.0 & 246 & 74.1 & & & \\
\hline Positive group & 426 & 43.0 & 86 & 25.9 & $5.9 \times 10^{-8}$ & 1.81 & $1.45-2.25$ \\
\hline
\end{tabular}

Table 2. Rate of PPD and MB in primiparas and multiparas. ${ }^{a}$ Edinburgh Postnatal Depression Scale (EPDS), The cut-off point of EPDS is 8/9, with scores of 9 or higher used to screen for minor and major depressive episodes. 'Stein's Maternity Blues Scale (Stein's Scale), The cut-off point of Stein's Scale is $7 / 8$, with scores of 8 or higher indicating mood swings or maternity blues. 'Adjusted $p$-value of the Bonferroni correction, EPDS: Bonferroni correction was performed by multiplying the number of test repetitions (4 time points) from $p$-value of chi-square test, Maternity blues: Bonferroni correction was performed by multiplying the number of test repetitions ( 5 time points) from $p$-value of chi-square test. ${ }^{\mathrm{d}}$ Adjusted $p$-value of the Bonferroni correction, Depressive group and Maternity blues: Bonferroni correction was performed multiplying the by number of test repetitions ( 2 factors) from $p$-value of chi-square test. T1, around 25 weeks of gestation; T2, around 36 weeks of gestation; T3, 5 days after delivery; T4, 1 month after delivery; Day1, 1 day after delivery; Day2, 2 days after delivery; Day3, 3 days after delivery; Day4, 4 days after delivery; Day5, 5 days after delivery; PPD, postpartum depression; $\mathrm{MB}$, maternity blues; $\mathrm{OR}$, odds ratio; $\mathrm{CI}$, confidence interval.

\begin{tabular}{|l|l|l|l|l|}
\hline \multicolumn{7}{|l|}{ n } & Mean (age) & \pm SD & Student's $\boldsymbol{t}$-test $\boldsymbol{p}$-value ${ }^{\mathbf{a}}$ \\
\hline Depressive group & 895 & 32.7 & \pm 4.5 & \\
\hline Non-depressive group & 138 & 32.7 & \pm 4.2 & 1.000 \\
\hline Postpartum depressive group & 818 & 32.6 & \pm 4.5 & \\
\hline Maternity blues & 524 & 32.2 & \pm 4.7 & 0.172 \\
\hline Negative group &
\end{tabular}

Table 3. Association of PPD and MB with age. PPD, postpartum depression; MB, maternity blues; SD, standard deviation. ${ }^{a}$ Adjusted $p$-value of the Bonferroni correction, Depressive group and Maternity blues: Bonferroni correction was performed multiplying the by number of test repetitions ( 2 factors) from $p$-value of Student's $t$-test. 


\begin{tabular}{|c|c|c|c|c|c|c|c|c|c|c|c|c|c|c|c|c|c|c|c|c|}
\hline & \multicolumn{5}{|l|}{ T1 } & \multicolumn{5}{|l|}{$\mathrm{T} 2$} & \multicolumn{5}{|l|}{ T3 } & \multicolumn{5}{|l|}{ T4 } \\
\hline & \multicolumn{2}{|c|}{ Primiparas } & \multicolumn{2}{|c|}{ Multiparas } & \multirow{2}{*}{\begin{tabular}{|l} 
ANCOVA $^{\mathrm{a}}$ \\
$p$-value \\
\end{tabular}} & \multicolumn{2}{|c|}{ Primiparas } & \multicolumn{2}{|c|}{ Multiparas } & \multirow{2}{*}{\begin{tabular}{|l|} 
ANCOVA $^{\text {a }}$ \\
$p$-value \\
b
\end{tabular}} & \multicolumn{2}{|c|}{ Primiparas } & \multicolumn{2}{|c|}{ Multiparas } & \multirow{2}{*}{$\begin{array}{l}\text { ANCOVA }^{a} \\
p \text {-value } \\
\end{array}$} & \multicolumn{2}{|c|}{ Primiparas } & \multicolumn{2}{|c|}{ Multiparas } & \multirow{2}{*}{\begin{tabular}{|l|} 
ANCOVA \\
$p$-value \\
\end{tabular}} \\
\hline & $\mathrm{N}$ & Mean \pm SD & $\mathrm{N}$ & Mean \pm SD & & $\mathrm{N}$ & Mean \pm SD & $\mathrm{N}$ & Mean $\pm S D$ & & $\mathrm{~N}$ & Mean \pm SD & $\mathrm{N}$ & Mean \pm SD & & $\mathrm{N}$ & Mean \pm SD & $\mathrm{N}$ & Mean \pm SD & \\
\hline Anxiety & 1129 & $1.9 \pm 1.7$ & 375 & $1.5 \pm 1.6$ & 0.011 & 1018 & $1.6 \pm 1.5$ & 341 & $1.3 \pm 1.5$ & 0.062 & 1016 & $1.5 \pm 1.6$ & 347 & $1.0 \pm 1.5$ & $4.8 \times 10^{-5}$ & 1031 & $1.6 \pm 1.6$ & 348 & $1.0 \pm 1.4$ & $9.2 \times 10^{-9}$ \\
\hline $\begin{array}{l}\text { Depres- } \\
\text { sion }\end{array}$ & 1128 & $0.5 \pm 1.0$ & 378 & $0.6 \pm 1.1$ & 1.000 & 1017 & $0.5 \pm 1.0$ & 342 & $0.6 \pm 1.0$ & 1.000 & 1015 & $0.6 \pm 1.0$ & 347 & $0.6 \pm 1.2$ & 1.000 & 1029 & $0.5 \pm 1.0$ & 347 & $0.4 \pm 0.9$ & 1.000 \\
\hline $\begin{array}{l}\text { Anhedo- } \\
\text { nia }\end{array}$ & 1129 & $0.3 \pm 0.8$ & 377 & $0.3 \pm 0.9$ & 1.000 & 1018 & $0.2 \pm 0.7$ & 340 & $0.2 \pm 0.7$ & 1.000 & 1018 & $0.4 \pm 0.9$ & 349 & $0.3 \pm 0.9$ & 1.000 & 1031 & $0.4 \pm 0.9$ & 348 & $0.3 \pm 0.8$ & 1.000 \\
\hline
\end{tabular}

Table 4. Comparison of EPDS factor scores between primiparas and multiparas from pregnancy to postpartum. T1, around 25 weeks of gestation; T2, around 36 weeks of gestation; T3, 5 days after delivery; T4, 1 month after delivery; SD, standard deviation; ANCOVA, analysis of covariance. ${ }^{a}$ In order to remove the effect of age, ANCOVA was performed with age as a covariate. ${ }^{\mathrm{b}}$ Adjusted $p$-value of the Bonferroni correction: Bonferroni correction was performed by multiplying the number of test repetitions $(3$ factors $\times 4$ time points $=12$ ) from $p$-value of ANCOVA.

\begin{tabular}{|l|l|l|l|l|l|l|}
\hline & \multicolumn{2}{l}{ Primiparas } & \multicolumn{1}{l|}{ Multiparas } & ANOCVA $^{\mathbf{a}}$ \\
\cline { 2 - 7 } & $\mathbf{n}$ & Mean \pm SD & n & Mean \pm SD & $\boldsymbol{p}^{\text {-value }}$ \\
\hline T1 J-SSQ & 1132 & $4.0 \pm 2.4$ & 378 & $3.5 \pm 1.6$ & 0.001 \\
\hline Number of persons & 1115 & $5.0 \pm 1.2$ & 369 & $4.8 \pm 1.1$ & 0.013 \\
\hline Satisfaction rating & 792 & $4.1 \pm 2.7$ & 206 & $3.5 \pm 1.6$ & 0.019 \\
\hline T4 J-SSQ & 779 & $4.9 \pm 1.3$ & 203 & $4.6 \pm 1.3$ & 0.009 \\
\hline Number of persons & & & & \\
\hline Satisfaction rating & & & & \\
\hline
\end{tabular}

Table 5. Comparison of J-SSQ scores in primiparas and multiparas. J-SSQ, the Japanese version of the Social Support Questionnaire; SD, standard deviation; ANCOVA, analysis of covariance; T1, around 25 weeks of

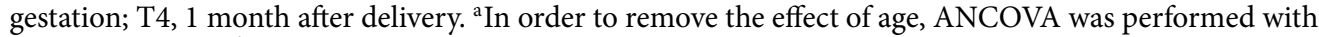
age as a covariate. ${ }^{b}$ Adjusted $p$-value of the Bonferroni correction: Bonferroni correction was performed by multiplying the number of test repetitions $(2$ factors $\times 2$ time points $=4)$ from $p$-value of ANCOVA.

\begin{tabular}{|c|c|c|c|c|c|}
\hline & \multicolumn{2}{|c|}{ Primiparas non-depressive group } & \multicolumn{2}{|c|}{$\begin{array}{l}\text { Primiparas postpartum depressive } \\
\text { group }\end{array}$} & \multirow{2}{*}{\begin{tabular}{|l|} 
ANCOVA $^{2}$ \\
$p$-value $^{\mathrm{b}}$
\end{tabular}} \\
\hline & $\mathbf{n}$ & Mean \pm SD & $\mathbf{n}$ & Mean \pm SD & \\
\hline \multicolumn{6}{|l|}{ T1 J-SSQ } \\
\hline Number of persons & 644 & $4.3 \pm 2.5$ & 114 & $3.9 \pm 2.1$ & 0.173 \\
\hline Satisfaction rating & 638 & $5.0 \pm 1.2$ & 111 & $5.0 \pm 1.1$ & 1.000 \\
\hline \multicolumn{6}{|l|}{ T4 J-SSQ } \\
\hline Number of persons & 485 & $4.4 \pm 3.1$ & 87 & $3.5 \pm 1.6$ & 0.011 \\
\hline Satisfaction rating & 477 & $4.9 \pm 1.3$ & 86 & $4.9 \pm 1.1$ & 1.000 \\
\hline
\end{tabular}

Table 6. J-SSQ scores in primiparas with and without PPD. J-SSQ, the Japanese version of the Social Support Questionnaire; PPD, postpartum depression; ANCOVA, analysis of covariance; SD, standard deviation; T1,

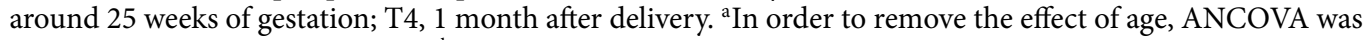
performed with age as a covariate. ${ }^{\mathrm{b}}$ Adjusted $p$-value of the Bonferroni correction: Bonferroni correction was performed by multiplying the number of test repetitions $(2$ factors $\times 2$ time points $=4)$ from $p$-value of ANCOVA.

factor for $\mathrm{PPD}^{10-12}$. We previously identified $\mathrm{MB}$ as a possible risk factor for $\mathrm{PPD}^{6}$, therefore, health care providers need to pay special attention to primiparas who present with MB.

There is a significant difference in average age between primiparas and multiparas, and there are reports that both being young ${ }^{30}$ and being old ${ }^{31}$ are risk factors for PPD. It has also been reported that becoming a mother at a young age is not itself a risk factor if it does not lead to a social disadvantage ${ }^{32}$. According to our results, it was parity, not age, that was associated with anxiety and PPD.

In this study, we found that primiparas had higher anxiety scores than multiparas, and in primiparas, the ratio of PPD was higher than in multiparas. Therefore, we need to be aware of anxiety in primiparas from early pregnancy. Primiparas probably have little child care experience and may lack self-confidence in their maternal role $^{26}$. It has been reported that primiparas have lower scores for maternal self-efficacy in neonatal care than do 
multiparas ${ }^{33}$. Less experience and a lack of confidence are likely to lead to anxiety. Therefore, the high level of anxiety about childbirth and childcare that primiparas experience for the first time would be not a pathological response but a natural one. However, some evidence that pregnancy and postpartum anxiety affect child development has been presented ${ }^{34}$. For example, it has been reported that maternal anxiety during early pregnancy has long-term associations with the cognitive development of the child to be born ${ }^{35}$. In addition, higher rates of behavioral and emotional problems have been reported in children whose mothers experienced high levels of anxiety in late pregnancy ${ }^{36}$. Further, maternal anxiety during pregnancy and the postpartum period have been reported to predict socio-emotional development ${ }^{37}$ and may be associated with developmental delays in children $^{38}$. Therefore, there is a need to cope with and reduce anxiety in primiparas. Increasing child care experience could be a promising way to prevent or mitigate PPD in primiparas ${ }^{39}$. Perinatal maternal anxiety exposure may lead to negative outcomes in child development, and more evidence is needed ${ }^{40}$.

When comparing primiparas and multiparas, the NP and SR subscale scores of the J-SSQ were higher in primiparas than in multiparas. Furthermore, it was revealed that in primiparas, the NP subscale score of the J-SSQ was significantly lower in the PPD group than in the non-depressive group. Studies on the quality of life of pregnant women have reported that being a primipara and having support from family and partner are associated with a better quality of life ${ }^{41}$. As noted in our previous study, social support can help prevent perinatal depression ${ }^{27}$. Moreover, a study of primiparas reported that most pregnant women recognize their partner, mother, or friends as the main provider of support ${ }^{42}$. Thus, informal support is very useful for the mental health of pregnant women, but many women in the perinatal period tend not to seek help in the face of mental disorders ${ }^{43}$. Therefore, it is important to provide pregnant women with information to help them increase the number of people who can provide them with support. In addition, it is important to inform pregnant women's family and friends that providing support is useful for the mental health of pregnant women. We are currently disseminating results of our research on perinatal mental health to pregnant women in perinatal classes, and are trying to provide information to their family and friends via a booklet about mental health that they can take home and use. It will be necessary to evaluate the effectiveness of these activities in a future study. There are also reports that expert support is useful for mothers who are at high risk of $\mathrm{PPD}^{44}$. Professional support may need to be considered for primiparas at a suspected high risk of PPD, such as those with low NP and SR subscale scores, those with high anxiety, and those experienced MB.

This study had several limitations. First, a selection bias was possible because the target facilities were limited to four hospitals. In addition, we could not include pregnant women who did not participate in the perinatal class. Second, the psychiatric variables used in this study, such as anxiety, depression, and anhedonia, were evaluated based on a self-reported questionnaire, not on a diagnosis by a psychiatrist. Third, the factors examined in this study were limited, as we did not examine mode of delivery or traumatic birth; such factors should be investigated in the future. Fourth, we only followed the participants until 1 month after delivery and could not confirm their progress over a longer term. In addition, we did not confirm the effects of anxiety and PPD on children. Long-term follow-up, including an investigation of these effects on children, is therefore necessary. In this study, we compared primiparas and multiparas, but since these groups had different members, they were independent of each other. In the future, it will be important to consider differences, such as the timing of first and second births, in the same woman.

\section{Conclusion}

The results of this study indicated that primiparas had higher rates of experiencing MB and higher rates of PPD than did multiparas. We also found that primiparas had higher anxiety scores since pregnancy than did multiparas, indicating that special attention needs to be paid to primiparas who present with MB. Primiparas had higher scores on the NP and SR subscales of the J-SSQ than did multiparas. However, when looking only at primiparas, the NP subscales score was lower in the PPD group than in the non-depressive group. From this result, it is considered that the number of supporters is more strongly associated with perinatal depression than is satisfaction with support. It is therefore important to provide pregnant women, especially primiparas, with information that increases the number of people who can provide them with support. In addition, professional support may need to be considered for primiparas with a suspected high risk of PPD, such as those with low NP and SR scores, high anxiety, and those who have experienced MB. The results of this study suggest that primiparas and multiparas have different characteristics, so it will be important to focus on parity in future studies.

\section{Methods}

Design. The data in the present study were extracted from a prospective cohort study conducted in Nagoya, Japan from August 2004 to March 2020 that included a self-administered questionnaire survey. The questionnaire included the EPDS, IDDL, J-SSQ, Stein's Scale, sociodemographic variables and questions about the baby's sex and weight. Regarding the sociodemographic variables, we explored age, years of education, presence or absence of mental disease, partner's age, and number of children. Among the sociodemographic questions and information on children, the age of the pregnant women and number of children were investigated from the beginning of the study in August 2004. We started collecting information about years of education, presence or absence of mental disease, partner's age, and baby's sex and weight in June 2012. The first questionnaire was completed in early pregnancy (T1: around 25 weeks of gestation) and included the EPDS, IDDL, J-SSQ, and sociodemographic questions. In late pregnancy (T2: around 36 weeks of gestation) and at 5 days after delivery (T3), the EPDS was completed again. On each of the first 5 days after delivery, Stein's Scale was completed. At 1 month after delivery (T4), the EPDS, J-SSQ, and questions about the baby were completed. At 1 month after delivery, the completed questionnaire was returned by postal mail. A flowchart of the study procedure is shown in Fig. 2. 
T1: around 25 weeks of gestation

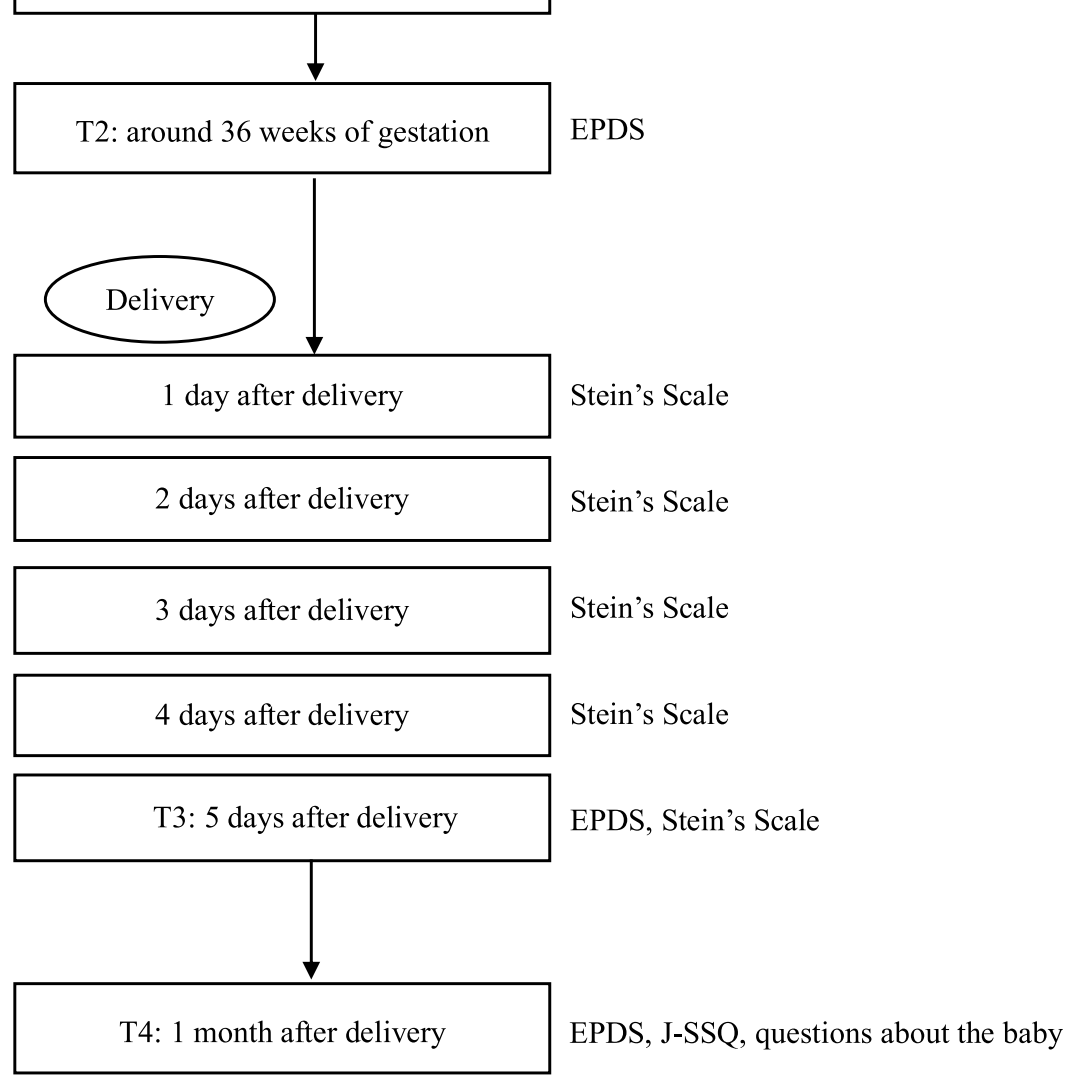

Figure 2. Flowchart of the study procedures. EPDS, Edinburgh Postnatal Depression Scale; IDDL, Inventory to Diagnose Depression, Lifetime version; J-SSQ, the Japanese version of the Social Support Questionnaire; Stein's Scale, Stein's Maternity Blues Scale.

Participants. Participants were recruited from one general hospital, two obstetrics and gynecology hospitals, and one university hospital. Pregnant women who were attending perinatal class that started before the 25 th week of pregnancy and who met the following eligibility criteria were asked to participate: (1) pregnant female aged 20 years or older, and (2) ability to read and write Japanese. The age of adulthood in Japan is 20 years. Therefore, in our study, we excluded pregnant women under the age of 20 years because they were considered minors.

Ethical considerations. A verbal and written explanation of the study was given to all participants, and written informed consent was obtained from all those who agreed to participate. The study protocol was approved by the Ethics Committee of the Nagoya University Graduate School of Medicine. All study methods met the Committee's guidelines and regulations and all study protocols were in accordance with the 1964 Helsinki Declaration and its later amendments or comparable ethical standards.

Measurements. EPDS. The EPDS is a self-administered questionnaire designed by Cox et al. in 1987 to screen for PPD ${ }^{45}$. The EPDS is comprised of 10 items and is scored on a four-point Likert scale (from 0 to 3 ), with total scores ranging from 0 to $30^{45}$. In our cohort study, we used the Japanese version of the EPDS, developed by Okano et al. in $1996^{46}$. Scores of $\geq 9$ are used to screen for minor and major depressive episodes (sensitivity: 75\% and $82 \%$, respectively; specificity: $93 \%$ and $95 \%$, respectively) ${ }^{23,46}$. In the present study, scores of $\geq 9$ were used to identify women with depression. In the original version of the EPDS, the cut-off point is $12 / 13^{45}$, but in the non-English version, a cut-off point lower than $12 / 13$ has been reported to be appropriate ${ }^{47-50}$. In this study, we used a cut-off point 8/9 for the Japanese version of the EPDS by Okano et al. ${ }^{46}$. The effectiveness of the EPDS as a screening scale for depression during pregnancy has also been reported ${ }^{51}$. The EPDS has three factors ("Anxiety", "Depression", and "Anhedonia"), and the factor structure has been demonstrated to be consistently stable throughout the peripartum period ${ }^{52}$.

We evaluated depressive symptoms using the EPDS during early pregnancy (around gestational week 25), during late pregnancy (around gestational week 36), at 5 days after delivery, and 1 month after birth. We categorized the participants into a "non-depressive group" or a "PPD group" based on EPDS score. Participants who scored under 8 points at the timepoints of approximately week 25, approximately week 36 , and 1 month after birth were categorized into the non-depressive group. Participants who scored under 8 points at the timepoints 
of around week 25 and around week 36 but scored over 9 points at 1 month postpartum were categorized into the PPD group.

IDDL. To assess the history of MDD among mothers, we used the IDDL. The IDDL is a self-administered questionnaire used to assess the history of MDD. It is based on the third version of the Diagnostic and Statistical Manual of Mental Disorders ${ }^{29}$ and comprises 22 items scored from 0 to 4 (5-point Likert scale). If a symptom continues for more than 2 weeks, the respondent is considered as having that symptom. A score of 2-4 indicates the presence of a specific symptom for each item, except items 5 and 6 , for which a score of $3-4$ is used. The conditions regarding a history of MDD were as follows for items that last 2 weeks or more: (1) five or more items scored at the cut-off score or higher, and (2) these five or more items contain at least one item related to the two major symptoms. Uehara et al. reported the sensitivity and specificity of the Japanese version of the IDDL to be $83 \%$ and $97 \%$, respectively ${ }^{53}$. We evaluated the history of MDD using the IDDL during early pregnancy (around week 25).

J-SSQ. The J-SSQ ${ }^{54}$ was used to measure social support among mothers in the present study. The J-SSQ has two factors, NP and $\mathrm{SR}^{27}$. NP is measured by the number subscale and reflects the average number of people the participant perceives as available to provide them with social support. SR is measured by the satisfaction subscale and reflects an individual's average degree of satisfaction with the perceived support available in various situations ${ }^{27}$. The J-SSQ has confirmed reliability and validity in the pregnancy and postpartum periods ${ }^{54}$. We evaluated social support using the J-SSQ during early pregnancy (around week 25) and at 1 month after birth. We started the evaluation of J-SSQ during early pregnancy in August 2004, and that at 1 month after birth in April 2006.

Stein's scale. Stein's Scale was used to assess early postpartum mood ${ }^{18}$. This scale has 13 items with total score ranging from 0 to 26 . The cut-off point is $7 / 8$, with scores of 8 or higher indicating mood swings or $\mathrm{MB}^{18}$. The Japanese version of Stein's Scale has proven validity and reliability for detecting MB in the early postpartum period $^{55}$. Since MB is transitory in nature, it is recommended that self-evaluation be conducted on consecutive days after delivery ${ }^{19}$. Therefore, mothers in this study completed Stein's Scale on each of the first 5 days after delivery. We categorized the participants into either a "negative group" or "positive group" based on Stein's Scale scores. The negative group scored under 8 points on each of the 5 days after delivery. The positive group scored over 8 points on one or more days after delivery. We evaluated MB using the Stein's Scale for 5 consecutive days (from the 1st to the 5 th day after delivery).

Statistical analyses. First, descriptive statistics based on the EPDS and J-SSQ were calculated. The listwise deletion method was used to handle missing data. All EPDS scores and J-SSQ subscale scores that were positively skewed were log-transformed in order to reduce the skewness. Then, the chi-square test, Student's $t$-test and ANCOVA were conducted to compare primiparas and multiparas, using the adjusted $p$-value from the Bonferroni correction. We considered adjusted $p$-values of $<0.05$ as significant.

\section{Data availability}

All the data supporting our findings are contained within the manuscript. Therefore, data sets are not shown. The raw data supporting the conclusions of this manuscript will be made available by the authors upon request.

Received: 18 February 2020; Accepted: 23 September 2020

Published online: 13 October 2020

\section{References}

1. Di Florio, A. et al. Perinatal episodes across the mood disorder spectrum. JAMA Psychiatry 70, 168-175 (2013).

2. O'Hara, M. W. \& Swain, A. M. Rates and risk of postpartum depression-a meta-analysis. Int. Rev. Psychiatry 8, 37-54 (1996).

3. Dietz, P. M. et al. Clinically identified maternal depression before, during, and after pregnancies ending in live births. Am. J. Psychiatry 164, 1515-1520 (2007).

4. Woody, C. A., Ferrari, A. J., Siskind, D. J., Whiteford, H. A. \& Harris, M. G. A systematic review and meta-regression of the prevalence and incidence of perinatal depression. J. Affect. Disord 219, 86-92 (2017).

5. O’Hara, M. W. \& Wisner, K. L. Perinatal mental illness: definition, description and aetiology. Best Pract. Res. Clin. Obstet. Gynaecol. 28, 3-12 (2014).

6. Ishikawa, N. et al. Prospective study of maternal depressive symptomatology among Japanese women. J. Psychosom. Res. 71, 264-269 (2011).

7. Liu, S. et al. Risk factors for postpartum depression among Chinese women: path model analysis. BMC Pregnancy Childbirth 17, 133 (2017).

8. el Saleh, S., El-Bahei, W., Del El-Hadidy, M. A. \& Zayed, A. Predictors of postpartum depression in a sample of Egyptian women. Neuropsychiatr. Dis. Treat. 9, 15-24 (2013).

9. Woolhouse, H., Gartland, D., Mensah, F. \& Brown, S. J. Maternal depression from early pregnancy to 4 years postpartum in a prospective pregnancy cohort study: implications for primary health care. BJOG 122, 312-321 (2015).

10. Takehara, K. et al. Prevalence trends of pre- and postnatal depression in Japanese women: a population-based longitudinal study. J. Affect. Disord. 225, 389-394 (2018).

11. Iwata, H. et al. Prevalence of postpartum depressive symptoms during the first 6 months postpartum: association with maternal age and parity. J. Affect. Disord. 203, 227-232 (2016).

12. Mori, T. et al. Psychosocial risk factors for postpartum depression and their relation to timing of onset: the Hamamatsu Birth Cohort (HBC) study. J. Affect. Disord. 135, 341-346 (2011).

13. Lara, M. A., Navarrete, L. \& Nieto, L. Prenatal predictors of postpartum depression and postpartum depressive symptoms in Mexican mothers: a longitudinal study. Arch. Womens Ment. Health 19, 825-834 (2016). 
14. Qandil, S., Jabr, S., Wagler, S. \& Collin, S. M. Postpartum depression in the occupied palestinian territory: a longitudinal study in Bethlehem. BMC Pregnancy Childbirth 16, 375 (2016).

15. Roomruangwong, C., Withayavanitchai, S. \& Maes, M. Antenatal and postnatal risk factors of postpartum depression symptoms in Thai women: a case-control study. Sex. Reprod. Healthc. 10, 25-31 (2016).

16. Hahn-Holbrook, J., Cornwell-Hinrichs, T. \& Anaya, I. Economic and health predictors of national postpartum depression prevalence: a systematic review, meta-analysis, and meta-regression of 291 studies from 56 countries. Front. Psychiatry 8, 248 (2017).

17. Pitt, B. Maternity blues. Br. J. Psychiatry 122, 431-433 (1973).

18. Stein, G. S. The pattern of mental change and body weight change in the first post-partum week. J. Psychosom. Res. 24, 165-171 (1980).

19. Kennerley, H. \& Gath, D. Maternity blues. I. Detection and measurement by questionnaire. Br. J. Psychiatry 155, 356-362 (1989).

20. O'Hara, M. W. \& McCabe, J. E. Postpartum depression: current status and future directions. Annu. Rev. Clin. Psychol. 9, 379-407 (2013).

21. Harris, B. et al. Maternity blues and major endocrine changes: cardiff puerperal mood and hormone study II. BMJ 308, 949-953 (1994).

22. Okano, T. \& Nomura, J. Endocrine study of the maternity blues. Prog. Neuropsychopharmacol. Biol. Psychiatry 16, 921-932 (1992).

23. Yamashita, H., Yoshida, K., Nakano, H. \& Tashiro, N. Postnatal depression in Japanese women. Detecting the early onset of postnatal depression by closely monitoring the postpartum mood. J. Affect. Disord. 58, 145-154 (2000).

24. Murata, A., Nadaoka, T., Morioka, Y., Oiji, A. \& Saito, H. Prevalence and background factors of maternity blues. Gynecol. Obstet. Invest. 46, 99-104 (1998).

25. Sakumoto, K., Masamoto, H. \& Kanazawa, K. Post-partum maternity "blues" as a reflection of newborn nursing care in Japan. Int. J. Gynaecol. Obstet. 78, 25-30 (2002).

26. Ferber, S. G. The nature of touch in mothers experiencing maternity blues: the contribution of parity. Early Hum. Dev. 79, 65-75 (2004).

27. Morikawa, M. et al. Relationship between social support during pregnancy and postpartum depressive state: a prospective cohort study. Sci. Rep. 5, 10520 (2015).

28. Shaw, E., Levitt, C., Wong, S., Kaczorowski, J. \& McMaster University Postpartum Research G. Systematic review of the literature on postpartum care: effectiveness of postpartum support to improve maternal parenting, mental health, quality of life, and physical health. Birth 33, 210-220 (2006).

29. Zimmerman, M. \& Coryell, W. The inventory to diagnose depression, lifetime version. Acta Psychiatr. Scand. 75, 495-499 (1987).

30. Zaidi, F., Nigam, A., Anjum, R. \& Agarwalla, R. Postpartum depression in women: a risk factor analysis. J. Clin. Diagn. Res. 11, QC13-QC16 (2017).

31. Matsumoto, K. et al. Age-specific 3-month cumulative incidence of postpartum depression: the Hamamatsu Birth Cohort (HBC) Study. J. Affect. Disord. 133, 607-610 (2011).

32. Schmied, V. et al. Maternal mental health in Australia and New Zealand: a review of longitudinal studies. Women Birth 26, 167-178 (2013).

33. Shorey, S., Chan, S. W., Chong, Y. S. \& He, H. G. Maternal parental self-efficacy in newborn care and social support needs in Singapore: a correlational study. J. Clin. Nurs. 23, 2272-2282 (2014).

34. Dennis, C. L., Falah-Hassani, K. \& Shiri, R. Prevalence of antenatal and postnatal anxiety: systematic review and meta-analysis. Br. J. Psychiatry 210, 315-323 (2017).

35. Van den Bergh, B. R. et al. High antenatal maternal anxiety is related to impulsivity during performance on cognitive tasks in 14- and 15-year-olds. Neurosci. Biobehav. Rev. 29, 259-269 (2005).

36. O'Connor, T. G., Heron, J., Golding, J., Glover, V. \& Team, A. S. Maternal antenatal anxiety and behavioural/emotional problems in children: a test of a programming hypothesis. J. Child Psychol. Psychiatry 44, 1025-1036 (2003).

37. Polte, C. et al. Impact of maternal perinatal anxiety on social-emotional development of 2-year-olds, a prospective study of norwegian mothers and their offspring: the impact of perinatal anxiety on child development. Matern. Child Health J. 23, 386-396 (2019).

38. Mughal, M. K. et al. Trajectories of maternal stress and anxiety from pregnancy to three years and child development at 3 years of age: findings from the All Our Families (AOF) pregnancy cohort. J. Affect. Disord. 234, 318-326 (2018).

39. Tsuchida, A. et al. Changes in the association between postpartum depression and mother-infant bonding by parity: longitudinal results from the Japan Environment and Children's Study. J. Psychiatr. Res. 110, 110-116 (2018).

40. Rees, S., Channon, S. \& Waters, C. S. The impact of maternal prenatal and postnatal anxiety on children's emotional problems: a systematic review. Eur. Child Adolesc. Psychiatry 28, 257-280 (2019).

41. Lagadec, N. et al. Factors influencing the quality of life of pregnant women: a systematic review. BMC Pregnancy Childbirth 18, 455 (2018)

42. Leahy-Warren, P., McCarthy, G. \& Corcoran, P. Postnatal depression in first-time mothers: prevalence and relationships between functional and structural social support at 6 and 12 weeks postpartum. Arch. Psychiatr. Nurs. 25, 174-184 (2011).

43. Liberto, T. L. Screening for depression and help-seeking in postpartum women during well-baby pediatric visits: an integrated review. J. Pediatr. Health Care 26, 109-117 (2012).

44. Kozinszky, Z. et al. Can a brief antepartum preventive group intervention help reduce postpartum depressive symptomatology? Psychother. Psychosom. 81, 98-107 (2012).

45. Cox, J. L., Holden, J. M. \& Sagovsky, R. Detection of postnatal depression. Development of the 10-item Edinburgh Postnatal Depression Scale. Br. J. Psychiatry 150, 782-786 (1987).

46. Okano, T. et al. Validation and reliability of Japanese version of the EPDS. Arch. Psychiatr. Diag. Clin. Eval. 7, 523-533 (1996).

47. Lee, D. T. et al. Detecting postnatal depression in Chinese women. Validation of the Chinese version of the Edinburgh postnatal depression scale. Br. J. Psychiatry 172, 433-437 (1998).

48. Jadresic, E., Araya, R. \& Jara, C. Validation of the Edinburgh Postnatal Depression Scale (EPDS) in Chilean postpartum women. J. Psychosom. Obstet. Gynaecol. 16, 187-191 (1995).

49. Vazquez, M. B. \& Miguez, M. C. Validation of the Edinburgh postnatal depression scale as a screening tool for depression in Spanish pregnant women. J. Affect. Disord. 246, 515-521 (2019).

50. Tesfaye, M., Hanlon, C., Wondimagegn, D. \& Alem, A. Detecting postnatal common mental disorders in Addis Ababa, Ethiopia: validation of the Edinburgh Postnatal Depression Scale and Kessler Scales. J. Affect. Disord. 122, 102-108 (2010).

51. Usuda, K., Nishi, D., Okazaki, E., Makino, M. \& Sano, Y. Optimal cut-off score of the Edinburgh Postnatal Depression Scale for major depressive episode during pregnancy in Japan. Psychiatry Clin. Neurosci. 71, 836-842 (2017).

52. Kubota, C. et al. Stable factor structure of the Edinburgh Postnatal Depression Scale during the whole peripartum period: Results from a Japanese prospective cohort study. Sci. Rep. 8, 17659 (2018).

53. Uehara, T., Sato, T., Sakado, K. \& Sato, S. The reliability and validity of the Japanese version of the inventory to diagnose depression, lifetime version (in Japanese). Arch. Psychiatr. Diag. Clin. Eval. 6, 73-81 (1995).

54. Furukawa, T. A., Harai, H., Hirai, T., Kitamura, T. \& Takahashi, K. Social support questionnaire among psychiatric patients with various diagnoses and normal controls. Soc. Psychiatry Psychiatr. Epidemiol. 34, 216-222 (1999).

55. Okano, T., Nomura, J., Koshikawa, N., Doi, M. \& Tatsunuma, T. Cross-cultural study of maternity blues and postpartum depression (in Japanese). Clin. Psychiatry 33, 1051-1058 (1991). 


\section{Acknowledgements}

We would like to thank the staff of Nagoya University Hospital, Nagoya Teishin Hospital, Kaseki Hospital, and Royal Bell Clinic for their cooperation.

\section{Author contributions}

Conception and design of the study: Y.N., T.O., M.A., and N.O.; acquisition and analysis of data: Y.N., T.O., M.M., A.Y., M.S., M.A., and N.O.; drafting the manuscript and tables: Y.N., T.O., and N.O.

\section{Funding}

This study was provided by research grants from the Ministry of Education, Culture, Sports, Science and Technology of Japan; the Ministry of Health, Labour and Welfare of Japan; the Academic Frontier Project for Private Universities, Comparative Cognitive Science Institutes, Meijo University; the Core Research for Evolutional Science and Technology; Intramural Research Grant (21B-2) for Neurological and Psychiatric Disorders from the National Center of Neurology and Psychiatry and the Specific Research Fund 2012 for East Japan Great Earthquake Revival by the New Technology Development Foundation; Research and Development Grants for Comprehensive Research for Persons with Disabilities from the Japan Agency for Medical Research and Development (AMED) under Grant Numbers JP20dk0307077; JSPS KAKENHI Grant Numbers JP20K07943. The funders had no role in the study design, data collection and analysis, decision to publish, or preparation of the manuscript.

\section{Competing interests}

The authors declare no competing interests.

\section{Additional information}

Correspondence and requests for materials should be addressed to T.O.

Reprints and permissions information is available at www.nature.com/reprints.

Publisher's note Springer Nature remains neutral with regard to jurisdictional claims in published maps and institutional affiliations.

(c) (i) Open Access This article is licensed under a Creative Commons Attribution 4.0 International License, which permits use, sharing, adaptation, distribution and reproduction in any medium or format, as long as you give appropriate credit to the original author(s) and the source, provide a link to the Creative Commons licence, and indicate if changes were made. The images or other third party material in this article are included in the article's Creative Commons licence, unless indicated otherwise in a credit line to the material. If material is not included in the article's Creative Commons licence and your intended use is not permitted by statutory regulation or exceeds the permitted use, you will need to obtain permission directly from the copyright holder. To view a copy of this licence, visit http://creativecommons.org/licenses/by/4.0/.

(c) The Author(s) 2020 